Please cite as:

Albó, L. Hernández-Leo, D. (2018). edCrumble: designing for learning with data analytics. Proceedings of the 13th European Conference on Technology Enhanced Learning, EC-TEL 2018, Leeds, UK, September 2018 (Accepted paper)

\title{
edCrumble: designing for learning with data analytics
}

\author{
Laia Albó ${ }^{[0000-0002-7568-9178]}$ and Davinia Hernández-Leo ${ }^{[0000-0003-0548-7455]}$ \\ ICT Department, Universitat Pompeu Fabra, Barcelona, Spain \\ \{laia.albo, davinia.hernadez-leo\}@upf.edu
}

\begin{abstract}
This demonstration introduces ILDE2/edCrumble, an online learning design platform that allows teachers the creation of learning designs (LDs) with the support of data analytics. ILDE2/edCrumble is built on top of the LdShake platform, which provides social features enabling the sharing and co-edition of LDs. The tool provides an innovative visual representation of LDs combining face-to-face and online learning in different places (in-class and out-of-class) and times (synchronous and asynchronous). Decision making during the LD process is supported by two types of analytics: resulting from the design of the activities sequenced in a timeline (LD analytics); and aggregated meta-data extracted from several grouped LDs (community analytics). Preliminary results conducted as part of an iterative design-based research process, show that the tool is being perceived as easy to use and useful. During the demo we will show the use case of how LD and community analytics can help balancing the workload and design between different courses which are part of a whole curriculum.
\end{abstract}

Keywords: Authoring tool, Learning Design, Data Analytics, Communities of educators, Visualization, Pedagogical planner, edCrumble, ILDE2, LdShake

\section{Introduction}

For some time now, Learning Design (LD) tools have been conceived to support teachers in the process of documenting their teaching practices, making their learning design ideas explicit and sharable [1][2][3][4]. The LD process often implies taking decisions about the selection of the most appropriate pedagogical model, the definition of the flow of tasks, the specification of roles as well as the choice of the most suitable resources and educational tools that can support the tasks defined, all to lead to potentially effective learning considering the needs of the educational context. However, despite existing proposed representations of pedagogical practice are varied, some are too specific for particular pedagogies and general approaches are not sufficiently accessible for teachers that do not have the required technical skills [5]. More intuitive visual representations of LD are needed [1][2]. Moreover, with the spread of ICTs more complex educational scenarios are arising -combining face-toface and online teaching in different places (in-class and out-of-class) and times (synchronous and asynchronous) [6]. [7] distinguishes two types of LD tools: "tools for visualizing designs" (which can be used to visualize and represent LDs) and the "pedagogical planners" (which can guide and support practitioners in making informed 
learning design decisions). In this paper, we present a LD tool that aims fitting in both categories bringing together the advantages of both types of tools. ILDE2/edCrumble can be considered a pedagogical planner which provides an innovative visual representation of the LDs characterized by data analytics with the aim of facilitating the planning, visualization, understanding and reuse of complex LDs. Specifically, the decision-making during the LD process is supported by two types of analytics [8]: resulting from the design of the activities sequenced in a timeline (LD analytics); and aggregated meta-data extracted from several grouped LDs created by multiple teachers within a community, e.g. a school (community analytics).

\section{Technological background}

edCrumble is a web-based running LD editor prototype developed in JavaScript and HTML5. It is mainly composed of five zones (see Fig.1), described as follows.

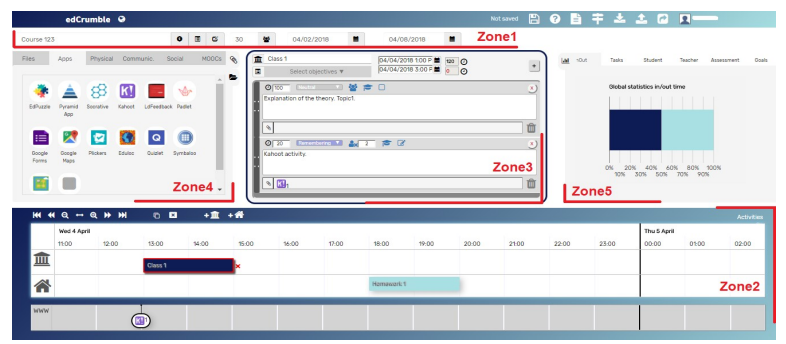

Fig. 1. edCrumble screenshot with the zones indicated in red (https://ilde2.upf.edu/edcrumble/)

Zone1: It allows users to provide general information about the LD. The title, number of students and the start and end dates of the LD. It has three buttons to specify: (a) the LD description, the educational level and topic; (2) the list of learning objectives; and (3) the evaluation. Zone2: It allows users to create in-class and out-of-class activities and place them in a timeline limited by the dates introduced in zone1. The timeline has two main layers by default (in and out-of-class), where the activities are visualized sequentially depending on their schedule and type. Zone3: It allows users to edit the activities. Once an activity is selected, the user can set up the corresponding learning objectives and add the tasks that compose it. Indicating and editing for each task: the time allocated, the cognitive process level associated (according to the Blooms' taxonomy [9]), the students type of work (individual, in groups or the whole class), the teacher's presence (teacher available face-to-face, online or not present), and the evaluation mode (graded task, not graded or task for auto-evaluation). The user can also write a description of the task to be done by the students with indicators for teachers and add the associated learning resources. Zone4: It allows users to select the resources for the activities. Resources are divided on different categories (placed in different tabs): Files, Apps, Physical, Communication, Social and MOOCs. The user can drag and drop a resource to the task of an activity and edit its characteristics: title, description, target (teacher or student resource), host-medium type (miscellanea, 
LMS, local storage, MOOC platform, web, physical artifact, cloud storage) and hostmedium name. Moreover, it is possible to specify an URL for the resource and/or upload a file. After adding a resource in an activity, a visualization of an icon associated to this resource appears automatically in the timeline, placed in a new layer depending on the host-medium type (see fig. 1 where a resource added in the second activity's task in zone3 appears in a host-medium layer -in grey- into the timeline in zone2, aligned with the corresponding activity). Zone5: It allows users to consult LD analytics extracted from the meta-data of the produced LD itself. Design analytics are divided on different categories (placed in different tabs): in-class/out-of-class time analytics, tasks 'cognitive process, student type of work, teacher presence, tasks' evaluation mode. In each category it is possible to have 3 different visualizations: global time statistics, statistics depending on the activities 'type (in or out-of-class) and depending on the learning objectives. Last, a button on the Zone2 allows users to have another view of the timeline hiding the time intervals between the activities and activating the analytics per activity (controlled by a legend composed by buttons corresponding to the different LD analytics' categories). Resulting in a completed interactive visual representation of the LD (see fig.2).

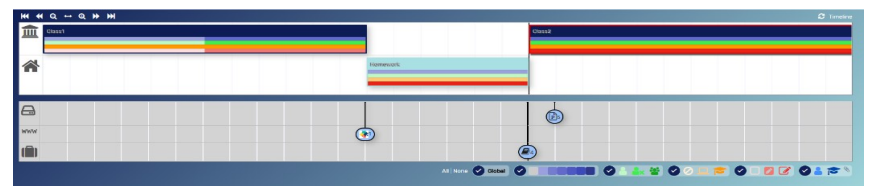

Fig. 2. Visual representation of a LD composed by 2 in-class and 1 out-of-class activities and 3 resources placed on 3 host-medium layers. Screenshot from the activities' analytics view.

edCrumble has been integrated as an authoring tool within the Integrated LD Environment (ILDE2) [4]. The integration of edCrumble into ILDE2 allows practitioners to co-edit, share, remix and comment their designs and others' designs within a community of teaching -ILDE2 is built on top of the LdShake platform that provides social network features [10]. Moreover, it facilitates teacher's access their designs for future design improvements during the iterative processes of the LD and teacher inquiry cycles (as LdShake acts as a repository of LDs). Once teachers have implemented their LDs, they can upload their evaluations to the edCrumble editor, helping others understand their impact and facilitating the adaptation and reusability of their LDs (for instance, describing the main challenges found or uploading links to the resulting learning analytics). The tool allows generating LD analytics aggregated from all the LDs placed in a folder, named as community analytics -supporting teachers' decision making during the LD process not only at their individual level but also allowing the possibility of considering the colleagues' LDs analytics in their community. The tool also offers the possibility of activating pedagogical guidelines (e.g. flipped classroom) during the design process as well as generating a LD summary including: (1) a printable syllabus with all the analytics generated; and (2) an interactive visualization to be embedded or shared with the colleagues but also with the students to help them organize their courses. 


\section{Use case, preliminary results and future work}

In the demo we will show the use case of how LD and community analytics extracted from ILDE2/edCrumble can help balancing the out-of-class workload between different courses which are part of a whole curriculum and support the necessary reflection process for specifically improving the LD quality of the activities within a community of educators. Despite the final evaluations of ILDE2/edCrumble are part of an ongoing cycle of a design-based research process, preliminary results from initial evaluation workshops with stakeholders indicate that the tool is being perceived as easy to use and useful. But also, the need for further work has been identified in the line of providing more flexibility during the activities' creation process (e.g. allowing users to import their activities from existing calendars or creating grouped activities which follow a certain time pattern).

Acknowledgements. This work has been partially funded by RecerCaixa (CoT project) and the Spanish Ministry of Economy and Competitiveness under MDM-20150502, TIN2014-53199-C3-3-R, TIN2017-85179-C3-3-R.

\section{References}

1. Conole, G. and Wills, S.: Representing learning designs - making design explicit and shareable. Educational Media International 50(1), 24-38 (2013).

2. Agostinho, S.: The use of a visual learning design representation to support the design process of teaching in higher education. Australas. J.Educ. Technol., 27(6), 961-978 (2011).

3. Laurillard, D. et al.: A constructionist learning environment for teachers to model learning designs. J. Comput. Assist. Learn., 29(1), 15-30 (2013).

4. Hernández-Leo, D., Asensio-Pérez, J. I., Derntl, M., Pozzi, F., Chacon-Perez, J., Prieto, L. P., \& Persico, D: An Integrated Environment for Learning Design. Frontiers in ICT, 5, 9 (2018).

5. Pozzi, F., Asensio-Pérez, J.I. and Persico, D.: The Case for Multiple Representations in the LD Life Cycle. The Future of Ubiquitous Learning. Springer, Berlin, Heidelberg (2016).

6. Norberg, A., Stöckel, M.B. Antti, M: Time Shifting and Agile Time Boxes in Course Design. International Review Of Research In Open \& Distributed Learning, 18(6) (2017).

7. Conole G.: Designing for learning in an open world, vol. 4. Springer Science \& Business Media (2012).

8. Hernández-Leo, D., Martinez-Maldonado, R., Pardo, A., Muñoz-Cristóbal, J. A., \& Rodríguez-Triana, M. J.: Analytics for learning design: A layered framework and tools. British Journal of Educational Technology (Accepted).

9. Krathwohl, D. R.: A revision of bloom's taxonomy: An overview. Theory Pract., 41(4), 212-218 (2002).

10.Hernández-Leo D. et al.: LdShake: Learning design solutions sharing and co-edition. Comput. Educ., 57(4), 2249-2260 (2011). 